\title{
Extreme value analysis of the time derivative of the horizontal magnetic field and computed electric field
}

\author{
Peter Wintoft $^{1}$, Ari Viljanen ${ }^{2}$, and Magnus Wik ${ }^{1}$ \\ ${ }^{1}$ Swedish Institute of Space Physics, Scheelevägen 17, 22370 Lund, Sweden \\ ${ }^{2}$ Finnish Meteorological Institute, Erik Palménin aukio 1, 00560 Helsinki, Finland \\ Correspondence to: Peter Wintoft (peter@lund.irf.se)
}

Received: 21 December 2015 - Revised: 11 April 2016 - Accepted: 18 April 2016 - Published: 2 May 2016

\begin{abstract}
High-frequency ( $\approx$ minutes) variability of ground magnetic fields is caused by ionospheric and magnetospheric processes driven by the changing solar wind. The varying magnetic fields induce electrical fields that cause currents to flow in man-made conductors like power grids and pipelines. Under extreme conditions the geomagnetically induced currents (GIC) may be harmful to the power grids. Increasing our understanding of the extreme events is thus important for solar-terrestrial science and space weather. In this work 1min resolution of the time derivative of measured local magnetic fields $\left(\left|\mathrm{d} \boldsymbol{B}_{h} / \mathrm{d} t\right|\right)$ and computed electrical fields $\left(\boldsymbol{E}_{h}\right)$, for locations in Europe, have been analysed with extreme value analysis (EVA). The EVA results in an estimate of the generalized extreme value probability distribution that is described by three parameters: location, width, and shape. The shape parameter controls the extreme behaviour. The stations cover geomagnetic latitudes from 40 to $70^{\circ} \mathrm{N}$. All stations included in the study have contiguous coverage of 18 years or more with 1-min resolution data. As expected, the EVA shows that the higher latitude stations have higher probability of large $\left|\mathrm{d} \boldsymbol{B}_{h} / \mathrm{d} t\right|$ and $\left|\boldsymbol{E}_{h}\right|$ compared to stations further south. However, the EVA also shows that the shape of the distribution changes with magnetic latitude. The high latitudes have distributions that fall off faster to zero than the low latitudes, and upward bounded distributions can not be ruled out. The transition occurs around $59-61^{\circ} \mathrm{N}$ magnetic latitudes. Thus, the EVA shows that the observed series north of $\approx 60^{\circ} \mathrm{N}$ have already measured values that are close to the expected maxima values, while stations south of $\approx^{\circ} \mathrm{N}$ will measure larger values in the future.
\end{abstract}

Keywords. Magnetospheric physics (auroral phenomena; magnetospheric configuration and dynamics; storms and substorms)

\section{Introduction}

Understanding the extreme conditions for the solar-terrestrial system is a scientific challenge and of importance to the society. Extreme conditions on the Sun result in powerful flares and fast coronal mass ejections (CMEs) (Cliver and Svalgaard, 2004) that may cause large disturbances on the geomagnetic field (Echer et al., 2008) with possible technological effects (Allen et al., 1989; Boteler and van Beek, 1993; Daglis, 2005; Gaunt, 2014).

The term extreme means that something far from the average or normal takes place, thus some level needs to be defined to be able to classify an event as extreme. The usual approach is to look at the distribution of events and set a limit at e.g. $0.1 \%$ percentiles and assuming that extreme events is the same as rare events.

The strongest geomagnetic storms are driven by fast CMEs with strong southward pointing magnetic field $\left(B_{z}\right)$, and particularly when multiple CMEs arrive at Earth. Fast Earth directed multiple halo CMEs produce the strongest geomagnetic storms as characterized by the Dst index (Gopalswamy et al., 2007).

Geomagnetic indices have an important role for solarterrestrial studies as they summarize some aspect of geomagnetic activity and they are generally global (Mayaud, 1980). Geomagnetic storms, in the general sense, display different temporal evolution depending on the choice of index. However, common to all storms are that they show a great range of variability in the geomagnetic field from periods less than a minute to several hours (Wintoft, 2005).

The driver of GIC is the geoelectric field (E) (Pirjola et al., 2004) which in turn is driven by the variations in the geomagnetic field (Viljanen et al., 2004). The high-frequency 
components of the geomagnetic field $(\lesssim$ minutes $)$ are the most important (Pulkkinen et al., 2006), but in cases with a less conductive layer above a highly conductive geological layer in the ground, the low-frequency components will have a large influence (Pirjola, 2010; Pulkkinen et al., 2010). However, for European conditions the magnitude of the calculated $\boldsymbol{E}$-field follows closely the magnitude of $\mathrm{d} \boldsymbol{B} / \mathrm{d} t$ (Wintoft et al., 2015). Thus, the total variation of the geomagnetic field is not the most important factor, but rather the time derivative $\mathrm{d} \boldsymbol{B} / \mathrm{d} t$ (Viljanen et al., 2001; Wintoft, 2005). The maximum geoelectric field can also be estimated from forecasts of maximum $\mathrm{d} \boldsymbol{B} / \mathrm{d} t$ (Wintoft et al., 2015).

The relevance of $\boldsymbol{E}$ or $\mathrm{d} \boldsymbol{B} / \mathrm{d} t$ to GIC has led to several studies related to their extreme behaviour (Wik et al., 2009; Myllys et al., 2014; Pulkkinen et al., 2015). In Thomson et al. (2011) extreme event analysis (EVA) was performed on $\mathrm{d} \boldsymbol{B} / \mathrm{d} t$ from stations covering similar latitudes as in this study, and 100- and 200-year return levels were estimated. The return level is obtained by extrapolating the data-fitted distribution to a small probability $p$ and interpreting that the corresponding level will be exceeded once every $1 / p$ years. The 100-year estimated return levels were found to lie in the range 1000-2000 $\mathrm{nT} \mathrm{min}^{-1}$ for geomagnetic latitudes above $60^{\circ} \mathrm{N}$ and typically below $1000 \mathrm{nT} \mathrm{min}^{-1}$ south of $53^{\circ} \mathrm{N}$. However, in the intermediate latitudes large values of 4000 $5000 \mathrm{nT} \mathrm{min}^{-1}$ were estimated. Sharp increases in amplitude of both $\mathrm{d} \boldsymbol{B} / \mathrm{d} t$ and calculated $\boldsymbol{E}$ around $50^{\circ} \mathrm{N}$ geomagnetic latitude were found by Pulkkinen et al. (2012) using data for the great storms of 13-15 March 1989 and 29-31 October 2003.

In this work 1-min resolution geomagnetic data and modelled electric field have been analysed using the generalized extreme value (GEV) distribution. As pointed out in Thomson et al. (2011) the generalized Pareto distribution (GPD) is appropriate when complete high-resolution measurement series are available as more data are used for the estimates. In GEV only one value is used out of the 500000 values in the year, but in GPD all values above a threshold are used. However, a threshold needs to be defined in the GPD and care need also to be taken for clustering so that only a single value from an event is included in the estimate. So in this respect the GEV is more general but at the same time fewer data points are considered. Another point is that the GEV used in this study forms an independent analysis against the GPD. Furthermore, we also apply the GEV analysis to the calculated electrical field at each site with measured magnetic fields.

\section{Analysis}

\subsection{Geomagnetic data and calculated electric field}

Geomagnetic data, at $1 \mathrm{~min}$ resolution, are obtained from World Data Centre for Geomagnetism (Edinburgh; http://

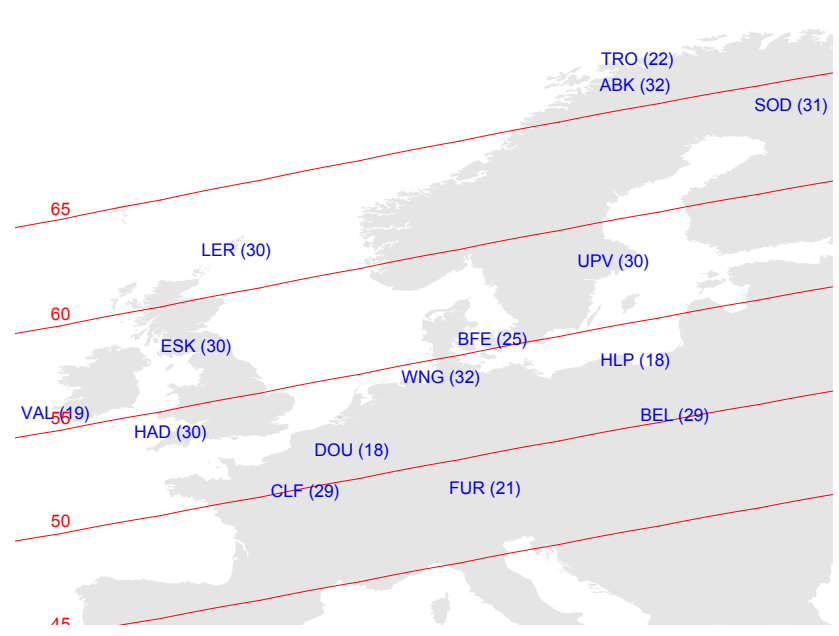

Figure 1. The locations of the magnetic observatories used. Each observatory has at least 18 years of contiguous 1-min data and the number of years of available data is shown in parenthesis. The red curves mark the geomagnetic latitudes, using the 11th generation IGRF coefficients, for epoch 1 January 2000.

www.wdc.bgs.ac.uk/catalog/master.html). The 1-min differences are formed as an approximation to the time derivative $\left(\mathrm{d} B_{x} / \mathrm{d} t, \mathrm{~d} B_{y} / \mathrm{d} t, \mathrm{~d} B_{z} / \mathrm{d} t\right)$ in the geographic $X-Y-Z$ coordinate system, where $X$ points north, $Y$ east, and $Z$ vertically down. The 15 stations included are shown on the map in Fig. 1. The criterium to include a station is that there should be at least 18 years of contiguous data. The limit of 18 years was set from a practical consideration to obtain a better coverage in central Europe, but in most cases there are close to 30 years of data or more. Naturally, the length of each series sets a limit on the confidence in the parameter estimations and return levels.

Each data set has also been cleaned from clearly erroneous measurements. In the $\mathrm{d} \boldsymbol{B} / \mathrm{d} t$ series they appear as sudden single spikes or double spikes with opposite signs, which in $\boldsymbol{B}$ corresponds to shifts or single spikes, respectively. The spikes occur during quiet times with low activity in nearby observatories. In addition, in the process of applying yearly block maxima (see next sections) each event from each station that caused the maximum can be further inspected as the total number of events is reasonably limited (between 18 to 32 events from each station).

The calculation of the electric field applies the method presented by Viljanen et al. (2012). It is based on the measured magnetic field and a local 1-D ground conductivity model specific for each observatory (Adam et al., 2012). Special attention is paid to missing values in the input magnetic field. We need a time series without data gaps for computing the electric field, so we have applied linear interpolation in case of missing magnetic field values. However, after the electric field has been determined, we have marked its values as missing at the time steps of originally missing magnetic field data. 


\subsection{Extreme value analysis - background}

We follow the approach described in Coles (2001) for our analysis. A time series $x(t)$ is divided into blocks of equal duration and then the maximum in each block is determined thereby producing a new series with block-maxima $z(t)$. If the number of values within each block-maxima is large, independent, and identically distributed then $z(t)$ will be distributed according to the generalized extreme value distribution

$G(z)=e^{-\left[1+\xi\left(\frac{z-\mu}{\sigma}\right)\right]^{-1 / \xi}}$,

where the probability that $z<z^{*}$ for some $z^{*}$ is $G\left(z^{*}\right)$, i.e. $\operatorname{Pr}\left\{z<z^{*}\right\}=G\left(z^{*}\right)$. The parameters $\mu, \sigma$, and $\xi$ are known as the location, width, and shape, respectively. The corresponding probability density function (PDF) is

$g(z)=\left[1+\xi\left(\frac{z-\mu}{\sigma}\right)\right]^{-(\xi+1) / \xi} e^{-\left[1+\xi\left(\frac{z-\mu}{\sigma}\right)\right]^{-1 / \xi}}$,

when $1+\xi\left(\frac{z-\mu}{\sigma}\right)>0$, else $g(z)=0$. In the special case with $\xi=0$ the $\mathrm{PDF}$ is

$g_{0}(z)=\frac{1}{\sigma} e^{-e^{-\frac{z-\mu}{\sigma}}-\frac{z-\mu}{\sigma}}$.

The shape $\xi$ is of special interest. If $\xi<0$, the PDF is zero for $z \geq \mu-\sigma / \xi$, i.e. $z$ has an upper limit. Note that $\mu$ and $\sigma$ are positive, therefore $\mu-\sigma / \xi$ is also positive for negative $\xi$. If instead $\xi \geq 0$, then $z$ has no upper limit, although $g(z) \rightarrow 0$ and $g_{0}(z) \rightarrow 0$ when $z \rightarrow \infty$. The larger the $\xi$ is, the slower $g(z)$ falls off to zero.

\subsection{Extreme value analysis - applied to $\mathrm{d} B / \mathrm{d} t$ and $E$}

From the observed horizontal magnetic field $\left[B_{x}(t ; s), B_{y}(t ; s)\right]$ at time $t$ and location $s$, the magnitude of its derivative is defined as

$x_{b}^{(s)}(t)=\sqrt{\left[\mathrm{d} B_{x}(t ; s) / \mathrm{d} t\right]^{2}+\left[\mathrm{d} B_{y}(t ; s) / \mathrm{d} t\right]^{2}}$.

As we are interested in the magnitude of the horizontal electric field, $|\boldsymbol{E}|=\sqrt{E_{x}^{2}+E_{y}^{2}}$, and that to a first approximation $\left(E_{x}, E_{y}\right) \propto\left(\mathrm{d} B_{y} / \mathrm{d} t,-\mathrm{d} B_{x} / \mathrm{d} t\right)$ we use the expression $\sqrt{\mathrm{d} B_{x} / t^{2}+\mathrm{d} B_{y} / t^{2}}$. This is different from taking the derivate of the horizontal magnetic field $\mathrm{d} B_{H} / \mathrm{d} t=\frac{d}{\mathrm{~d} t} \sqrt{B_{x}^{2}+B_{y}^{2}}$. Similarly the magnitude of the calculated horizontal electric field vector $\left[E_{x}(t ; s), E_{y}(t ; s)\right]$ is

$x_{e}^{(s)}(t)=\sqrt{E_{x}^{2}(t ; s)+E_{y}^{2}(t ; s)}$.

Block-maxima $z_{b}^{(s)}(t)$ and $z_{e}^{(s)}(t)$ are calculated from $x_{b}^{(s)}(t)$ and $x_{e}^{(s)}(t)$, respectively. The length of each block

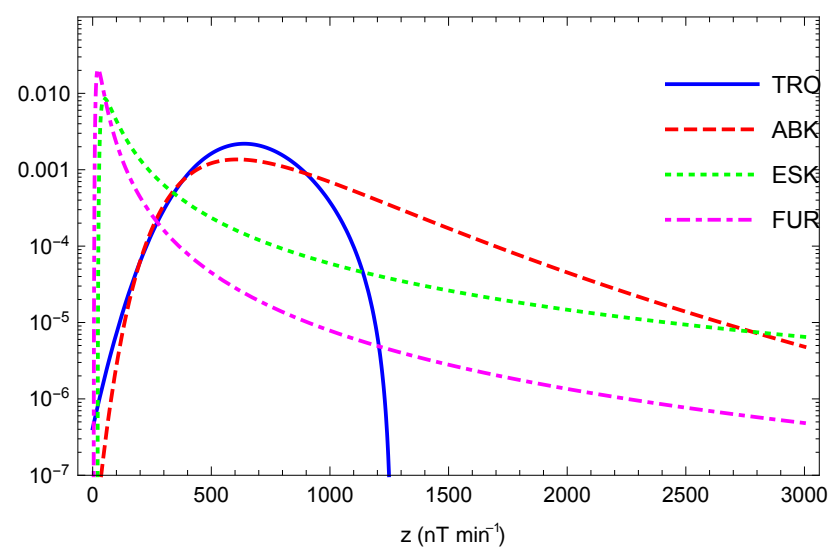

Figure 2. Probability density functions for four distributions estimated from geomagnetic data at TRO, ABK, ESK, and FUR.

should be long enough to contain many samples, but at the same time the block-maxima series $z$ should also be as long as possible for the statistical interpretation. From a theoretical viewpoint the observations within a block should be independent and identically distributed (i.i.d.). Clearly, it is difficult to show that i.i.d. holds in the strict sense, however, we believe it is a reasonable assumption according to the following. We choose yearly block-maxima as they contain a large number of values. Although 1 year consists of about 526000 1-min values, they are not independent. Considering individual substorms one may argue independence on timescales of hours. However, substorms may also be part of a storm and then the timescales are of the order of hours to a few days. Thus, each yearly block maxima contains at least $\sim 100$ (days timescale) to thousands (hours timescale) of independent values. Finally, we assume that they are identically distributed (i.d.), which naturally can be questioned. However, the i.d. assumption is to some degree supported by the result by Weigel and Baker (2003) who found that the form of the distribution of large $\mathrm{d} \boldsymbol{B} / \mathrm{d} t$ for a high-latitude station (SOD) was independent of the solar wind forcing, time of day, and day of year.

The three parameters $\mu, \sigma$, and $\xi$ are found by maximizing the log-likelihood function using the block-maxima series $z(t)$. The results are shown for each station in Table 1 . As expected, the location $\mu$ and width $\sigma$ generally increase with increasing magnetic latitude. However, the shape $\xi$ is generally smaller, and even negative, at high magnetic latitudes. The estimated distributions for the stations TRO, ABK, ESK, and FUR are shown in Fig. 2. Notice that the estimated distribution at TRO has an upper limit of the block-maxima $z$ due to the negative $\xi$. It is also seen that the ABK distribution falls off faster then the ESK and FUR distributions.

As the shape parameter $\xi$ is especially interesting for the extreme behaviour the parameter interval at $95 \%$ confidence level is estimated. As a first approximation it can be assumed that $\xi$ is normally distributed leading to a straightforward es- 
Table 1. The magnetic latitude, number of years $(N)$, maximum of block-maxima $z\left(\mathrm{nT} \min ^{-1}\right)$, the estimated parameters $\mu\left(\mathrm{nT}\right.$ min $\left.{ }^{-1}\right), \sigma$ $\left(\mathrm{nT} \min ^{-1}\right)$, and $\xi$ for each analysed station. Note that UPV is the combined series from the LOV and UPS stations. The magnetic latitudes have been calculated using the 11th generation IGRF coefficients for year 2000.

\begin{tabular}{lrrrrrr|rrrrr}
\hline & & \multicolumn{3}{c}{$\mathrm{d} \boldsymbol{B} / \mathrm{d} t\left(\mathrm{nT} \mathrm{min}^{-1}\right)$} & \multicolumn{4}{c}{$E\left(\mathrm{mV} \mathrm{km}^{-1}\right)$} \\
\cline { 3 - 11 } Station & M. lat. & $N$ & $\max z$ & $\mu$ & $\sigma$ & $\xi$ & $\max z$ & $\mu$ & $\sigma$ & $\xi$ \\
\hline TRO & 67.0 & 22 & 1050 & 589 & 174 & -0.26 & 1994 & 1398 & 383 & -0.60 \\
ABK & 65.9 & 32 & 2248 & 640 & 272 & 0.12 & 4081 & 1409 & 570 & 0.09 \\
SOD & 63.7 & 31 & 1997 & 462 & 192 & 0.18 & 3297 & 1082 & 419 & 0.11 \\
LER & 61.9 & 30 & 992 & 204 & 142 & 0.05 & 2430 & 530 & 361 & 0.12 \\
UPV & 58.0 & 30 & 2688 & 135 & 133 & 0.69 & 1938 & 160 & 153 & 0.60 \\
ESK & 57.7 & 30 & 1271 & 81 & 62 & 0.97 & 2412 & 174 & 126 & 0.80 \\
VAL & 55.7 & 19 & 358 & 34 & 23 & 0.70 & 645 & 89 & 53 & 0.63 \\
BFE & 55.3 & 25 & 1995 & 96 & 95 & 1.10 & 1562 & 121 & 95 & 0.82 \\
WNG & 54.0 & 32 & 716 & 61 & 50 & 0.90 & 172 & 23 & 15 & 0.54 \\
HAD & 53.8 & 30 & 327 & 40 & 26 & 0.91 & 703 & 121 & 67 & 0.46 \\
HLP & 53.0 & 18 & 326 & 56 & 37 & 0.73 & 198 & 52 & 29 & 0.31 \\
DOU & 51.3 & 18 & 261 & 35 & 21 & 0.69 & 416 & 64 & 36 & 0.74 \\
BEL & 50.0 & 29 & 219 & 48 & 31 & 0.57 & 277 & 59 & 30 & 0.39 \\
CLF & 49.7 & 29 & 312 & 37 & 24 & 0.62 & 480 & 85 & 49 & 0.54 \\
FUR & 48.2 & 21 & 196 & 32 & 21 & 0.65 & 703 & 121 & 76 & 0.58 \\
\hline
\end{tabular}

timate of the confidence interval. The covariance matrix is computed from the sum of the Hessian evaluated at each $z_{i}$ given the estimated GEV distribution. The square root of the diagonal terms are then multiplied by the inverse normal cumulative distribution at the $95 \%$ level. However, a more accurate approach is to base the confidence interval on the profile of the log-likelihood function

$$
\begin{aligned}
\ell(\mu, \sigma, \xi)= & -m \log \sigma-(1+1 / \xi) \sum_{i=1}^{m} \log \left[1+\xi\left(\frac{z_{i}-\mu}{\sigma}\right)\right] \\
& -\sum_{i=1}^{m}\left[1+\xi\left(\frac{z_{i}-\mu}{\sigma}\right)\right]^{-1 / \xi} .
\end{aligned}
$$

The maximum of $\ell$, denoted by $\ell^{*}$, is obtained for each station by the parameters in Table 1. For the ESK station the maximum is $\ell^{*}=-186.9$ as seen in Fig. 3. The profile of the $\log$-likelihood function is achieved by iteratively changing $\xi$ away from its optimal value by small amounts and finding new optimal values for $\mu$ and $\sigma$ for which $\ell$ is locally maximized. The $95 \%$ level is reached when $2\left(\ell^{*}-\ell(\mu, \sigma, \xi)\right)<$ $c_{0.05}$, where $c_{0.05}$ is the $1-0.05=0.95$ quantile of the $\chi_{1}^{2}$ distribution (Coles, 2001). Figure 3 shows the profile for the ESK station, and the limits obtained both from the profile and from assuming a normal distribution. It is seen that the profile limits are asymmetric around the log-likelihood maximum and shifted towards larger values as compared to the normal limits.

The shapes $\xi$, determined from $|\mathrm{d} \boldsymbol{B} / \mathrm{d} t|$ and $|\boldsymbol{E}|$, at the different locations are shown in Fig. 4 with respect to the magnetic latitude. The profile estimates of the confidence limits at $95 \%$ confidence level are indicated with the vertical



Figure 3. Profile log-likelihood for the ESK station. Likelihood values at maximum and $95 \%$ confidence level are indicated by horizontal dashed lines. The vertical dashed lines indicate the limits of $\xi$ obtained from the profile. The dotted lines indicate the limits obtained by assuming a normal distribution.

bars. All stations below magnetic $58^{\circ} \mathrm{N}$ have positive values for $\xi$ determined from $|\mathrm{d} \boldsymbol{B} / \mathrm{d} t|$ at the $95 \%$ confidence level, while stations above $61^{\circ} \mathrm{N}$ contain both positive and negative $\xi$. The variation of the widths of the confidence limits are caused by a couple of factors. The length of each series has some influence, however, in this case not large, as the number of samples ranges from 18 to 32 and the confidence limits are approximately inversely proportional to the square root of the sample size. There is a also a "natural" variation as samples are randomly drawn from the underlying distribution. Finally, samples from a distribution with positive $\xi$ will 


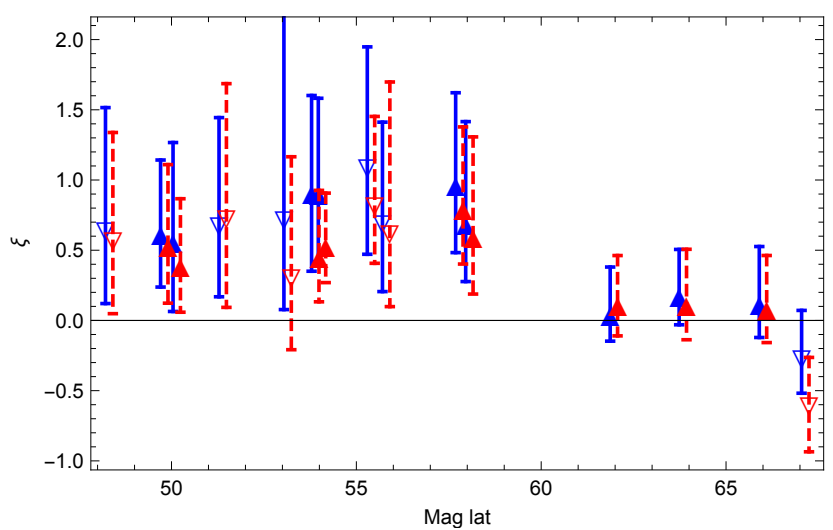

Figure 4. The figure shows the shape $\xi$ as function of magnetic latitude for $|\mathrm{d} \boldsymbol{B} / \mathrm{d} t|$ (blue, solid) and $E$ (red, dashed). Bars indicate error limits at $95 \%$ confidence level estimated from the profile. Filled upward pointing triangles and unfilled downward pointing triangles mark stations with more than and less than 25 years of data, respectively.

be more spread out than samples coming from a distribution with $\xi$ around zero, see e.g. Fig. 2. Therefore, there will generally be a larger uncertainty associated with positive $\xi$ than with $\xi$ around zero, hence the narrower confidence intervals at higher latitudes.

The $\xi$ parameter estimated from the calculated electric field shows a similar variation with latitude. Stations above $\sim 60^{\circ} \mathrm{N}$ lie around $\xi=0$, while stations south of that have positive $\xi$, with one exception, namely the HLP station. However, it should be noted that there are only 18 years of data from the HLP station (Fig. 1).

Although all stations selected have data extending over 18 years or more, they cover slightly different time intervals. For example, the UPV station includes the year 1982 with an extreme of $2688 \mathrm{nT} \mathrm{min}^{-1}$, while some of the other stations do not include that year. To test the effect on the estimate of the distribution parameters we remove the data for that year and redo the analysis for UPV. The result yields $(\mu, \sigma, \xi)=(137,125,0.49)$ which is similar to that in Table 1 . The new confidence limits also puts $\xi$ above zero, with lower and upper $95 \%$ confidence limits of $\xi$ equal to $(0.09$, 1.19).

From the distributions the 100-year return levels can be estimated. We find that the return levels are similar in magnitude and latitudinal variation as found by Thomson et al. (2011). However, in this analysis the $95 \%$ confidence limits are much larger making it hard to come to a strong conclusion.

We also turned the question around and asked what is the return period for some high level. Selecting a value of $3000 \mathrm{nTmin}^{-1}$ it is above all observed maxima (Table 1) but not to a great extent from that observed at $A B K$ and UPV. The fitted distribution at TRO will never reach $3000 \mathrm{nT} \mathrm{min}^{-1}$ as $\xi$ is less than zero. The ABK, SOD,
Table 2. The observed maxima (Obs. max) and the return periods, in years, for the different stations to levels of observed maxima (Max), two times observed maxima (2 Max), and fixed $3000 \mathrm{nT} \mathrm{min}^{-1}$ level (3000).

\begin{tabular}{lrrrr}
\hline Station & $\begin{array}{r}\text { Obs. max } \\
\left.(\mathrm{nT} \mathrm{min})^{-1}\right)\end{array}$ & $\begin{array}{r}\text { Max } \\
\text { (Years) }\end{array}$ & $\begin{array}{r}2 \text { Max } \\
\text { (Years) }\end{array}$ & $\begin{array}{r}3000 \\
(\text { Years })\end{array}$ \\
\hline ABK & 2248 & 86 & 3700 & 370 \\
SOD & 1997 & 140 & 3200 & 840 \\
LER & 992 & 140 & 20000 & 1200000 \\
UPV & 2688 & 47 & 130 & 55 \\
ESK & 1271 & 22 & 44 & 53 \\
VAL & 358 & 31 & 84 & 650 \\
BFE & 1995 & 18 & 33 & 26 \\
WNG & 716 & 17 & 37 & 84 \\
HAD & 327 & 14 & 31 & 160 \\
HLP & 326 & 13 & 34 & 280 \\
DOU & 261 & 23 & 62 & 780 \\
BEL & 219 & 13 & 42 & 1200 \\
CLF & 312 & 29 & 87 & 1100 \\
FUR & 196 & 17 & 47 & 1100 \\
\hline
\end{tabular}

and LER stations have an average $\bar{\xi}=0.1$ and their corresponding $3000 \mathrm{nT} \mathrm{min}^{-1}$ return periods are much larger than the lengths of the corresponding measurements series as shown in Table 2. The mid-latitude stations (UPV, ESK, BFE, WNG) have $\bar{\xi}=0.9$ and $3000 \mathrm{nT} \mathrm{min}^{-1}$ return periods in the range 30-80 years, except the VAL station. The lower latitude stations (HAD, HLP, DOU, BEL, CLF, FUR) have $\bar{\xi}=0.7$ and return periods in the range $200-1000$ years. The VAL station has a very low maximum $|\mathrm{d} \boldsymbol{B} / \mathrm{d} t|$, in accordance with Thomson et al. (2011), in that both predicted and observed extremes are significantly less compared to stations at similar magnetic latitudes. However, the $\xi$ parameter is still positive at the $95 \%$ significance level.

Clearly, with return periods largely exceeding the lengths of the analysed time series the uncertainty is very large. E.g. the LER station, with $10^{6}$ year return period at $3000 \mathrm{nT} \mathrm{min}^{-1}$, is caused by a small $\xi$ and that the observed maximum of $992 \mathrm{nT} \mathrm{min}^{-1}$ is far from the $3000 \mathrm{nT} \mathrm{min}{ }^{-1}$ limit. In particular, the LER $3000 \mathrm{nT} \mathrm{min}^{-1}$ return period can be brought down to below 800 years by changing its $\xi$ within the $95 \%$ confidence limits. This is also in line with the 200year return levels shown in Fig. 6 by Thomson et al. (2011) when comparing with $\mathrm{ABK}$ and SOD.

Further, we may also relate the return periods to limits determined from the individual station maxima in Table 2. The high latitude stations have return periods longer than the extent of the measured series for reaching the currently observed maxima. The more southernly stations generally have return periods similar to or shorter than the observed series lengths.

We may also explore the effect on $\xi$ on the chosen conductivity model. From Table 1 it is seen that $\xi$ for all stations, 
except two, have lower values for $|\boldsymbol{E}|$ than for $|\mathrm{d} \boldsymbol{B} / \mathrm{d} t|$, i.e. $\xi_{E}<\xi_{\mathrm{d} \boldsymbol{B} / \mathrm{d} t}$. A simple frequency-independent scaling is not sufficient to change the values of $\xi$. This is an effect of that in $\mathrm{d} \boldsymbol{B} / \mathrm{d} t$ only the high-frequency components of $\boldsymbol{B}$ are considered, while in $\boldsymbol{E}$ there is also some contribution from the lower-frequency components in $\boldsymbol{B}$. To test this we chose the SOD station and changed the conductivity of the deep layer. The original model has two layers: a top layer of $30 \mathrm{~km}$ depth with resistivity $5000 \Omega \mathrm{m}$ and an infinitely deep bottom layer with $200 \Omega \mathrm{m}$ (Adam et al., 2012). The bottom layer resistivity is then changed to $20 \Omega \mathrm{m}$ (same as UPV) and $2 \Omega \mathrm{m}$ and $\xi$ recalculated. The damping of the high-frequency components results in that $\xi_{E}$ is decreased from 0.11 to 0.099 and 0.091 , respectively.

\section{Conclusions}

Extreme value analysis (EVA) has been applied to 1-min resolution time series of locally measured $\mathrm{d} \boldsymbol{B} / \mathrm{d} t$ for 14 stations in Europe (Fig. 1), and for locally calculated $\boldsymbol{E}$. The higher latitude stations have on average higher levels of disturbance as shown by the location $(\mu)$ and width $(\sigma)$ (Table 1$)$. This is expected due to the stations proximity to the auroral oval.

For the shape parameter $\xi$ the results show that, at $95 \%$ confidence level, stations south of $\approx 60^{\circ} \mathrm{N}$ have positive shape parameter $\xi$, indicating upwardly unbounded distributions. On the other hand, stations north of $\approx 60^{\circ} \mathrm{N}$ have $\xi$ values close to zero, and negative values can not be ruled out. If the results hold, it has implications on what levels of disturbance can be achieved in the extreme cases. Assuming that $\xi$ is negative for the northern stations (TRO, ABK, SOD, LER) then the maximum $|\mathrm{d} \boldsymbol{B} / \mathrm{d} t|$ or $|\boldsymbol{E}|$ have upper limits and values not much higher than what has already been observed are anticipated (see maximum values in Table 1). For the other stations the $\xi$ parameters are larger and definitively positive and thus the maximum observed values will be exceeded in the future. But even if it is assumed that the northerly stations have positive $\xi$, the distribution still falls off quicker than for more lower latitude stations (Fig. 2).

From Table 1 it is seen that $\xi$ for all stations, except two, have lower values for $|\boldsymbol{E}|$ than for $|\mathrm{d} \boldsymbol{B} / \mathrm{d} t|$, i.e. $\xi_{E}<\xi_{\mathrm{d} \boldsymbol{B} / \mathrm{d} t}$, an effect of that in $\mathrm{d} \boldsymbol{B} / \mathrm{d} t$ only the high-frequency components of $\boldsymbol{B}$ are considered, while in $\boldsymbol{E}$ there is also some contribution from the lower-frequency components in $\boldsymbol{B}$. This was also confirmed at one location (SOD). In future work it would be interesting to further explore the $\xi$ dependency on the conductivity model, and also especially try to understand why $\xi_{E}$ is larger than $\xi_{\mathrm{d} \boldsymbol{B} / \mathrm{d} t}$ for some locations.

The return periods for a $3000 \mathrm{nT} \mathrm{min}^{-1}$ disturbance were computed from the fitted distributions, except for TRO which can never reach that level. The three high latitude stations showed return periods of several hundred years or more, similar to the most southernly stations, while the mid-latitude stations showed $30-80$ years return periods. It is generally stated that geomagnetic variations are most intense at high latitudes. This is true when considering, for example, longterm averages of field variations. However, it is does not automatically follow that the same holds for extreme events. As observations of the July 1982, March 1989 and October 2003 storms indicate, magnetic variations and $\mathrm{d} \boldsymbol{B} / \mathrm{d} t$ reached values at subauroral latitudes that were comparable or even larger than at the average auroral region (Kappenman, 2005, 2006; Pulkkinen et al., 2005; Wik et al., 2009). Observations also show that ionospheric electrojets move southwards when their intensity increases (Ahn et al., 2005; Rostoker and Duc Phan, 1986). Thus, the physical interpretation could be as follows. During moderate-size storms the auroral oval extends over higher latitudes and therefore the high latitude stations will observe larger $\mathrm{d} \boldsymbol{B} / \mathrm{d} t$ compared to stations at lower latitudes. However, during the extreme storms the oval will move further southward with the result that the more extreme $\mathrm{d} \boldsymbol{B} / \mathrm{d} t$ will be observed further south.

Acknowledgements. This work has been funded by the following grants: European Community's Seventh Framework Programme (FP7/2007-2013) under Grant Agreement No. 260330; and Swedish Civil Contingencies Agency, contract MSB-2011-4186. We thank WDC-Edinburgh and IMAGE, and the contributing institutes, for providing geomagnetic observatory data in Europe. We would also like to thank the reviewers (J. M. Torta and an anonymous) for improving the manuscript with valuable comments.

The topical editor, G. Balasis, thanks J. M. Torta and one anonymous referee for help in evaluating this paper.

\section{References}

Adam, A., Pracser, E., and Wesztergom, V.: Estimation of the electric resistivity distribution (EURHOM) in the European lithosphere in the frame of the EURISGIC WP2 project, Acta Geod. Geoph. Hung., 47, 377-387, doi:10.1556/AGeod.47.2012.4.1, 2012.

Ahn, B. H., Chen, G. X., Sun, W., Gjerloev, J. W., Kamide, Y., Sigwarth, J. B., and Frank, L. A.: Equatorward expansion of the westward electrojet during magnetically disturbed periods, J. Geophys. Res., 110, A01305, doi:10.1029/2004JA010553, 2005.

Allen, J., Frank, L., Sauer, H., and Reiff, P.: Effects of the March 1989 solar activity, Eos Transactions AGU, 70, 1989.

Boteler, D. H. and van Beek, G. J.: Mapping the March 13, 1989, magnetic disturbance and its consequences across North America, in: Solar-Terrestrial Predictions-IV, edited by: Hruska, J., Shea, M. A., Smart, D. F., and Heckman, G., Geological Survey of Canada, Contribution Series 37692, Vol. 3, 57-70, 1993.

Cliver, E. W. and Svalgaard, L.: The 1859 solar-terrestrial disturbance and the current limits of extreme space weather activity, Solar Phys., 224, 407-422, 2004.

Coles, S.: An Introduction to Statistical Modeling of Extreme Values, Springer Series in Statistics, Springer-Verlag London, 2001.

Daglis, I. A.: Effects of Space Weather on Technology Infrastructure, NATO Science Series II: Mathematics, Physics and Chem- 
istry, Springer-Verlag Berlin Heidelberg, doi:10.1007/1-40202754-0, 2005.

Echer, E., Gonzalez, W. D., Tsurutani, B. T., and Gonzalez, A. L. C.: Interplanetary conditions causing intense geomagnetic storms (Dst $\leq-100 \mathrm{nT}$ ) during solar cycle 23 (1996-2006), J. Geophys. Res., 113, A05221, doi:10.1029/2007JA012744, 2008.

Gaunt, C. T.: Reducing uncertainty - responses for electricity utilities to severe solar storms, J. Space Weather Space Clim., 4, A01, doi:10.1051/swsc/2013058, 2014.

Gopalswamy, N., Yashiro, S., and Akiyama, S.: Geoeffectiveness of halo coronal mass ejections, J. Geophys. Res., 112, A06112, doi:10.1029/2006JA012149, 2007.

Kappenman, J. G.: An overview of the impulsive geomagnetic field disturbances and power grid impacts associated with the violent Sun-Earth connection events of 29-31 October 2003 and a comparative evaluation with other contemporary storms, Space Weather, 3, S08C01, doi:10.1029/2004SW000128, 2005.

Kappenman, J. G.: Great geomagnetic storms and extreme impulsive geomagnetic field disturbance events - An analysis of observational evidence including the great storm of May 1921, Adv. Space Res., 38, 188-199, 2006.

Mayaud, P. N.: Derivation, meaning, and use of geomagnetic indices, Geophysical monograph, Vol. 22, American Geophysical Union, 1980.

Myllys, M., Viljanen, A., Rui, Ø. A., and Ohnstad, T. M.: Geomagnetically induced currents in Norway: the northernmost highvoltage power grid in the world, J. Space Weather Space Clim., 4, A10, doi:10.1051/swsc/2014007, 2014.

Pirjola, R.: Derivation of characteristics of the relation between geomagnetic and geoelectric variation fields from the surface impedance for a two-layer earth, Earth Planet. Space, 62, 287295, 2010.

Pirjola, R., Viljanen, A., Pulkkinen, A., Kilpua, S., and Amm, O.: Ground effects of space weather, Space weather effects on electric power transmission grids and pipelines, NATO Science Series, Kluwer Academic Publishers, 2004.

Pulkkinen, A., Lindahl, S., Viljanen, A., and Pirjola, R.: October 29-31, 2003 geomagnetic storm: geomagnetically induced currents and their relation to problems in the Swedish highvoltage power transmission system, Space Weather, 3, S08C03, doi:10.1029/2004SW000123, 2005.

Pulkkinen, A., Viljanen, A., and Pirjola, R.: Estimation of geomagnetically induced current levels from different input data, Space Weather, 4, S08005, doi:10.1029/2006SW000229, 2006.

Pulkkinen, A., Kataoka, R., Watari, S., and Ichiki, M.: Modeling geomagnetically induced currents in Hokkaido, Japan. Adv. Space Res., 46, 1087-1093, 2010.
Pulkkinen, A., Bernabeu, E., Eichner, J., Beggan, C., and Thomson, A. W. P.: Generation of 100-year geomagnetically induced current scenarios, Space Weather, 10, S04003, doi:10.1029/2011SW000750, 2012.

Pulkkinen, A., Bernabeu, E., Eichner, J., Viljanen, A., and $\mathrm{Ng}$ wira, C.: Regional-scale high-latitude extreme geoelectric fields pertaining to geomagnetically induced currents, Earth Planets Space, 69, 93, doi:10.1186/s40623-015-0255-6, 2015.

Rostoker, G. and Duc Phan, T.: Variation of auroral electrojet spatial location as a function of the level of magnetospheric activity, J. Geophys. Res., 91, 1716-1722, doi:10.1029/JA091iA02p01716, 1986.

Thomson, A., Dawson, E. B., and Reay, S. J.: Quantifying extreme behavior in geomagnetic activity, Space Weather, 9, S100001, doi:10.1029/2011SW000696, 2011.

Viljanen, A., Nevanlinna, H., Pajunpää, K., and Pulkkinen, A.: Time derivative of the horizontal geomagnetic field as an activity indicator, Ann. Geophys., 19, 1107-1118, doi:10.5194/angeo-191107-2001, 2001

Viljanen, A., Pulkkinen, A., Amm, O., Pirjola, R., Korja, T., and BEAR Working Group: Fast computation of the geoelectric field using the method of elementary current systems and planar Earth models, Ann. Geophys., 22, 101-113, doi:10.5194/angeo-22101-2004, 2004

Viljanen, A., Pirjola, R., Wik, M., Ádám, A., Prácser, E., Sakharov, Y., and Katkalov, Y.: Continental scale modelling of geomagnetically induced currents, J. Space Weather Space Clim., 2, A17, doi:10.1051/swsc/2012017, 2012.

Weigel, R. S. and Baker, D. N.: Probability distribution invariance of 1-minute auroral-zone geomagnetic field fluctuations, Geophys. Res. Lett., 30, 2193, doi:10.1029/2003GL018470, 2003.

Wik, M., Pirjola, R., Lundstedt, H., Viljanen, A., Wintoft, P., and Pulkkinen, A.: Space weather events in July 1982 and October 2003 and the effects of geomagnetically induced currents on Swedish technical systems, Ann. Geophys., 27, 1775-1787, doi:10.5194/angeo-27-1775-2009, 2009.

Wintoft, P.: Study of the solar wind coupling to the time difference horizontal geomagnetic field, Ann. Geophys., 23, 1949-1957, doi:10.5194/angeo-23-1949-2005, 2005

Wintoft, P., Wik, M., and Viljanen, A.: Solar wind driven empirical forecast models of the time derivative of the ground magnetic field, J. Space Weather Space Clim., 5, A7, doi:10.1051/swsc/2015008, 2015. 